\title{
Non-local fringe image filtration: a new interferometric data filtration paradigm?
}

\author{
Maciej Wielgus ${ }^{1,2^{*}}$ and Krzysztof Patorski ${ }^{1}$ \\ ${ }^{1}$ Institute of Micromechanics and Photonics, Warsaw University of Technology, Sw A. Boboli 8, 02-525 Warszawa \\ ${ }^{2}$ Institute of Electron Technology, Al. Lotników 32/46, 02-668 Warszawa
}

Received May 18, 2012; accepted June 20, 2012; published June 30, 2012

\begin{abstract}
In this letter we discuss the notion of non-local filtration in the context of fringe pattern images processing, to contrast it with commonly used denoising techniques restricted to the close neighborhood of a given pixel. We evaluate the performance of the nonlocal means algorithm on exemplary fringe patterns to compare it with several other methods and show filtration efficiency influence on phase calculation quality.
\end{abstract}

Noise is an inevitable part of any measurement, not excluding interferometric fringe pattern image recording. It can be introduced by environment, electronic hardware or even be an intristic part of the image formation process, as in the case of speckle noise. Suppressing a random noise component from the acquired data is crucial to enable accurate determination of the measured quantity, being the fringe phase in most interferometry applications. If the data are registered digitally, metrology can benefit from advanced computer-aided processing techniques aimed at noise suppression. A large number of algorithms were proposed both for the general image and specific fringe pattern denoising. Although these algorithms do vary significantly, one may find common paradigms for most, if not all of them:

1. Random noise can be removed by some kind of averaging (typically: change pixel intensity value to an adjacent pixels intensity mean value, possibly weighted);

2. Averaging is spatially local in nature, as we can reasonably assume that intensity values of a sought unspoiled image are locally similar, allowing to average out the random component.

The question to be answered is whether significantly more efficient fringe pattern denoising algorithms can be developed if we do not limit ourselves to a local neighborhood, denying the second paradigm. In particular, local averaging results in resolution losses and this shortcoming can be overcome by non-local filtration techniques. Note that non-local methods can be considered particularly interesting for fringe pattern processing.

*E-mail: maciek.wielgus@gmail.com
This is because the fringe pattern possesses a quasiperiodic structure and a quasi-periodic (or, at least, not strictly decreasing with distance) autocorrelation function, i.e., very similar pixels can be separated by a certain distance.

Although the notion of combining truly similar pixels rather than just neighboring pixels was present in image processing for a long time [1], a satisfying and explicit formulation of the problem was not given until 2005 [2, 3 ], when the non local means (NLM) algorithm was presented. In the NLM approach, the estimation $\mathbf{u}^{\mathbf{E}}$ of the true intensity value distribution $\mathbf{u}^{*}$ is calculated from noise spoiled initial data $\mathbf{v}$ by averaging values of many pixels with weights based on their neighborhood similarity.

$$
u^{E}(i)=\sum_{j \in \Omega} w(i, j) v(j)
$$

where $\mathrm{i}, \mathrm{j}$ represent the pixels from image domain $\Omega$. A Gaussian weighted Eucildean distance $\|\cdot\|_{\sigma}$ can be used as a similarity measure between neighborhoods $\mathrm{N}_{\mathrm{i}}$ and $\mathrm{N}_{\mathrm{j}}$ of pixels $\mathrm{i}$ and $\mathrm{j}$. The proposed wages are given by

$$
w(i, j)=\frac{1}{C(i)} \exp \left(-\frac{\left\|v\left(N_{i}\right)-v\left(N_{j}\right)\right\|_{\sigma}^{2}}{h^{2}}\right)
$$

with normalization $\mathrm{C}(\mathrm{i})$

$$
C(i)=\sum_{j \in \Omega} \exp \left(-\frac{\left\|v\left(N_{i}\right)-v\left(N_{j}\right)\right\|_{\sigma}^{2}}{h^{2}}\right) .
$$

The motivation to use the exponential function of a negative argument is that it is smooth, decreasing and of value equal to one for the argument equal to zero. Parameter $h$ represents the filtration strength, being stronger for higher values of $h$. Clearly, the distance between pixels $i$ and $j$ is not taken into account in weight calculations, which are based solely on corresponding neighborhood similarity. Therefore pixels from any part of the image can contribute to the final evaluation $\mathbf{u}^{\mathbf{E}}$, at 
least, theoretically, as for practical reasons the area of search can be constricted to improve the algorithm time performance.

The NLM algorithm was originally employed to the photographic image enhancement, further method developments were reported in the field of ultrasound [4] and SAR (Synthetic Aperture Radar) [5] images processing. Just recently, a non-local method similar to NLM has been introduced to the fringe pattern analysis in [6].

In Fig. 1 (a) a synthetic fringe pattern is shown. We spoil it with a Gaussian noise, SNR $=3$, Fig. 1 (b). We evaluate the performance of several denoising methods in Figs. 1 (c-f). These are a simple linear Gaussian filter, median filter, adaptive Wiener filter, continuous wavelet transform (CWT) filter, Perona-Malik filter based on nonlinear diffusion, and NLM algorithm. All the algorithms were tuned, and their parameters were chosen to optimize the performance. Small windows had to be used for median, Gaussian and Wiener filters as very dense fringes were present in the central region of the image. Clearly, the NLM outperforms other methods. Quantitative comparison results are given in Table 1. The comparison of the (normalized) mean square error (MSE) shows that NLM has even much greater ability of reducing noise than advanced methods such as nonlinear diffusion filtering. It is worth noticing that filtration based on CWT (2D Morlet wavelet in this case) performs highly satisfactorily in the regions of quasi-straight fringes. However, at the image borders and in the regions of large fringe curvature CWT performs poorly, see the bottom left corner of Fig. 1 (f).

The parameters used for the NLM algorithm were the size of neighborhoods used for comparisons, which was chosen to be $7 \times 7$ pixels, whereas the filtration strength parameter $h=0.5$. We limited the search window radius to 20 pixels for time performance optimalization reasons.

Denoising efficiency has a direct influence on the quality of further fringe pattern processing results. We treated denoised images with BEMD-HT procedure for pattern demodulation, as described in $[7,8]$. The method is based on Bidimensional Empirical Mode Decomposition (BEMD) algorithm and Hilbert transform (HT). BEMD allows to isolate the oscillatory component of the image effectively. Such a component is subsequently processed with the Hilbert transform or some kind of its 2D generalization. In these investigations, particularly, a vortex transform [9] was used for that purpose. BEMDHT is a robust demodulation algorithm, capable, in most cases, of retrieving the phase with a small error. Only the regions of very low fringe density or large fringe curvature represent a problematic task. With BEMD-HT we calculated the wrapped phase distribution for each image presented in Fig. 1 (b-f) and compared it with a wrapped original phase, see Table 1 . The last row shows the MSE of BEMD-HT demodulation.

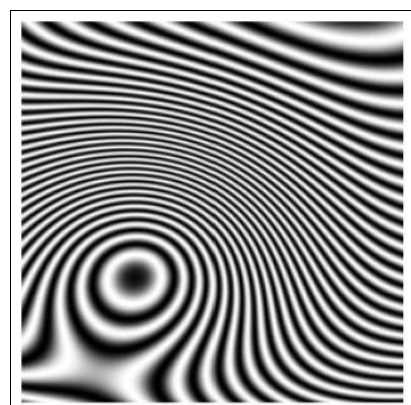

(a)

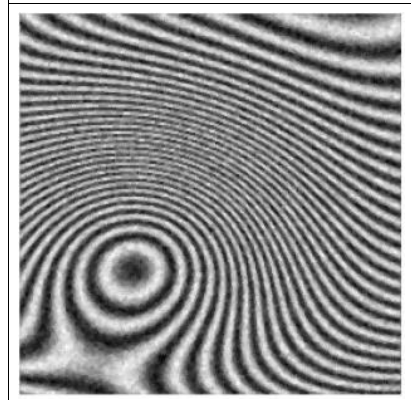

(c)

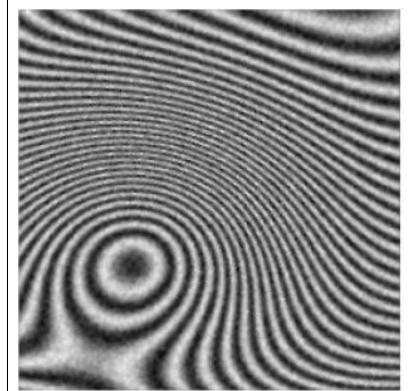

(e)

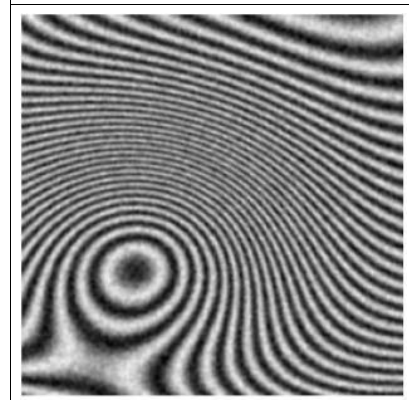

(g)

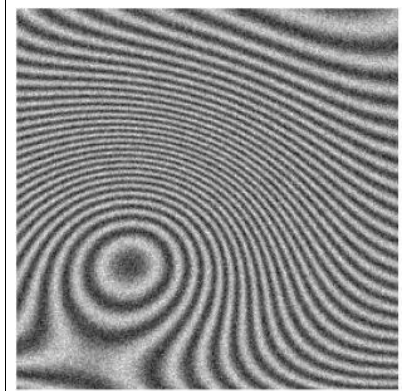

(b)

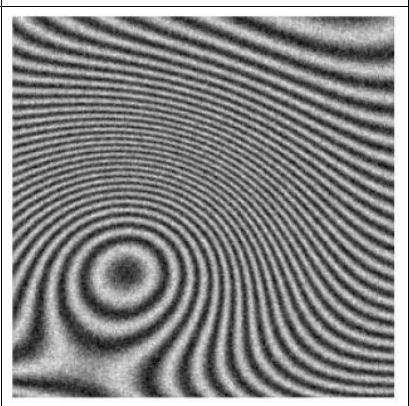

(d)

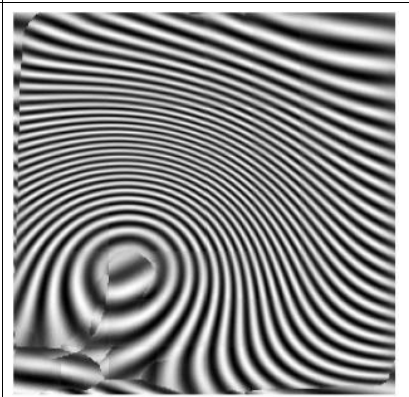

(f)

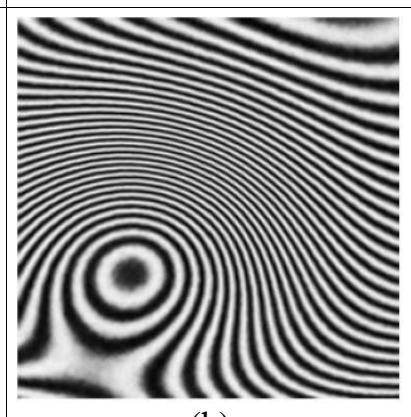

(h)
Fig. 1. Synthetic fringe pattern (a); synthetic fringe pattern with noise (b); filtration result with median (c), Gaussian (d), Wiener (e), CWTbased (f), Perona-Malik (g) and NLM filter (h).

The data given in Table 1 confirm a significant influence of noise on phase calculation quality, justifying the efforts to develop more efficient denoising procedures. More than $70 \%$ of noise influence on phase calculations was removed by filtration with the NLM algorithm. 
Further analysis demonstrations are given in Figs. 2-3. Phase error distributions (right columns, range limited to $\pi / 9>\Delta \varphi>\pi / 36$ ) display a large random component present in the results. Only the phase obtained with NLM shows an error originating mostly from the demodulation method, see the resemblance between Fig. 2 (b) and Fig. 3 (d).

Table 1. Methods efficiency comparison

\begin{tabular}{|l|l|l|l|l|}
\hline & MSE & $\begin{array}{l}\text { MSE } \\
\text { reduction }\end{array}$ & $\begin{array}{l}\text { Phase } \\
\text { MSE }\end{array}$ & $\begin{array}{l}\text { Noise error } \\
\text { reduction }\end{array}$ \\
\hline Noised & 0.425 & $0 \%$ & 0.661 & $0 \%$ \\
\hline Median & 0.310 & $26,9 \%$ & 0.496 & $35.5 \%$ \\
\hline Gauss & 0.288 & $32.9 \%$ & 0.490 & $36.8 \%$ \\
\hline Wiener & 0.288 & $32.9 \%$ & 0.426 & $50.6 \%$ \\
\hline CWT & 0.462 & - & 0.944 & - \\
\hline $\begin{array}{l}\text { Perona- } \\
\text { Malik }\end{array}$ & 0.255 & $40.0 \%$ & 0.398 & $56.5 \%$ \\
\hline NLM & 0.159 & $62.5 \%$ & 0.318 & $73.9 \%$ \\
\hline No noise & 0 & $100 \%$ & 0.197 & $100 \%$ \\
\hline
\end{tabular}

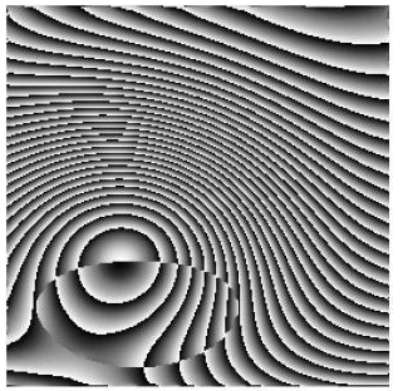

(a)

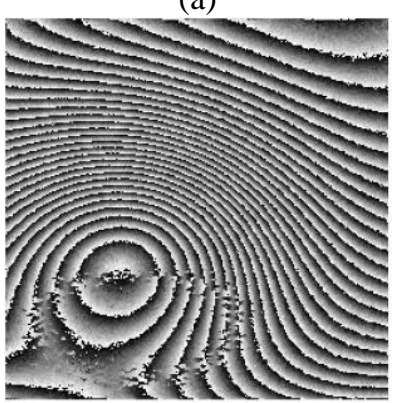

(c)

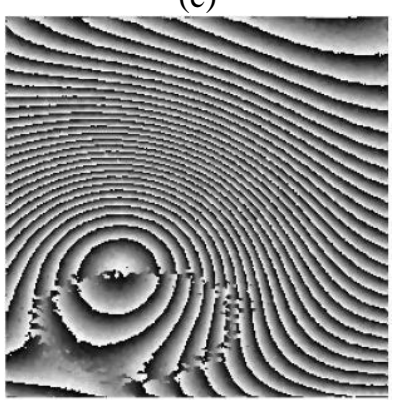

(e)

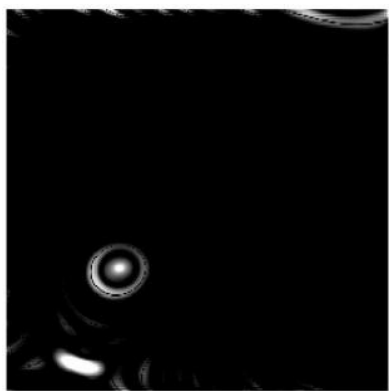

(b)

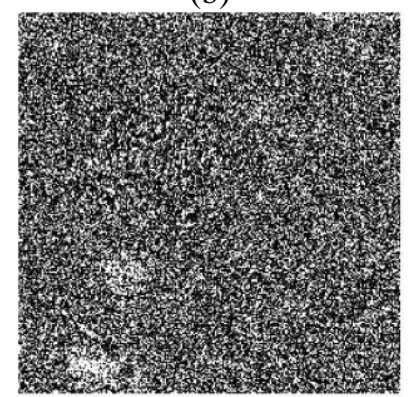

(d)

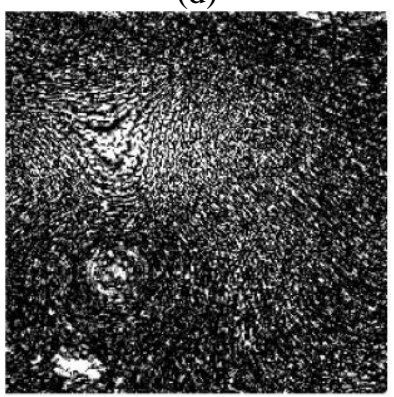

(f)
Fig. 2. Wrapped phase mod $2 \pi$ and phase error distributions for: unspoiled image (a-b); noised image without filtration (c-d); median filtration (e-f).
The effect of a phase sign change visible in the bottom left corner of wrapped phase distributions is the result of fringe orientation angle ambiguity and can be removed easily with a priori information of phase distribution.

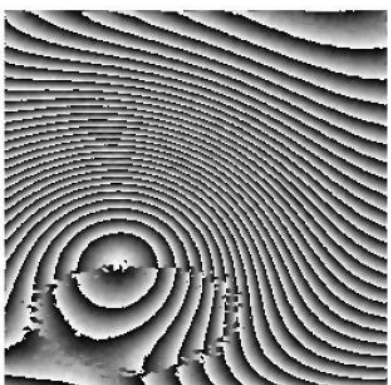

(a)

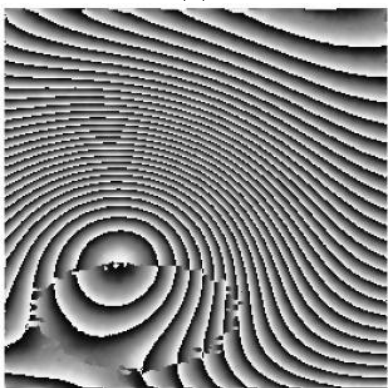

(c)

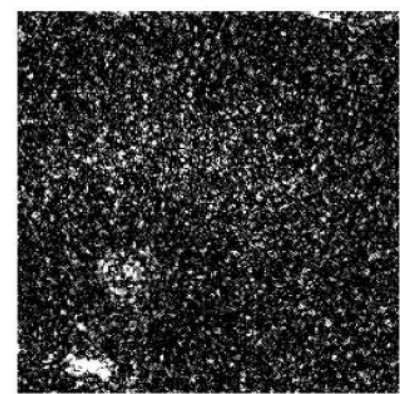

(b)

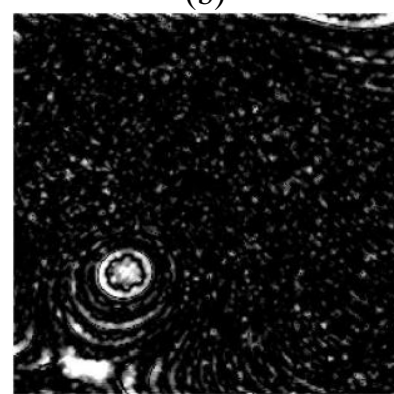

(d)
Fig. 3. Wrapped phase $\bmod 2 \pi$ and phase error distributions for: Perona-Malik filtration (a-b); NLM filtration (c-d).

The presented results indicate that the non-local filtration method applied to fringe pattern image is capable of producing high quality denoising results. Moreover, more sophisticated algorithms based on NLM can substantially benefit from the knowledge of noise distribution. Further development of such algorithms may constitute an important step in data digital processing for optical metrology.

\section{References}

[1] L. Yaroslavsky, Digital Picture Processing - An Introduction (Springer Verlag, 1985).

[2] A. Buades, B. Coll, J.M. Morel, Proc. IEEE CVPR (2005).

[3] A. Buades, B. Coll, J.M. Morel, Int. J. of Comp. Vision 76, 123 (2008).

[4] P. Coupe, P. Hellier, C. Kervrann, C. Barillot, IEEE Trans. Image Proc. 18, 10 (2009).

[5] C.A. Deledalle, L. Denis, F. Tupin, IEEE Trans. on Geosc. Remote Sens. 49, 4 (2011).

[6] S. Fu, C. Zhang, Opt. Commun. 285, 2541(2012).

[7] M. Bernini, A. Federico, G. Kaufmann, Appl. Opt. 50, 641 (2011).

[8] M. Wielgus, K. Patorski. Appl. Opt. 50, 5513 (2011).

[9] K.G. Larkin, D.J. Bone, M.A. Oldfield, J. Opt. Soc. Am. 18, 1871 (2001). 